\title{
Proceeding
}

Supplementary Issue: Rio 2016 Olympic Games Third Anniversary Special Edition. Olympic Studies Forum, 2-3 September 2019. Federal University of Sergipe, Aracaju, Brazil

\section{Do executive functions discriminate sports performance in young soccer athletes?}

\section{As funções executivas discriminam 0 desempenho esportivo em jovens atletas de futebol?}

\author{
RODRIGO FLORES SARTORI ${ }^{1,2} \triangle$, DANIEL MEZAVILLA², GLAUBER CARVALHO NOBRE ${ }^{3}$, ROCHELE $^{2}$ \\ PAZ FONSECA 4 \\ ${ }^{1}$ Department of Physical Education, Pontifical Catholic University of Rio Grande do Sul, Brazil \\ 2University of Serra Gaúcha - FSG, Brazil \\ ${ }^{3}$ Department of Physical Education, Federal Institute of Education, Science and Technology, Ceará, Brazil \\ ${ }^{4}$ Department of Psychology, Pontifical Catholic University of Rio Grande do Sul, Brazil
}

\begin{abstract}
Executive funtions are important for good soccer performance, requiring adaptation to changing environment and quick anticipation of your actions. In our study we sought to compare the ability to design executive functions in soccer athletes and their schoolmates (non-athletes) in a total of eighty pre-adolescents aged 11 and 12 years. The following components were evaluated; inhibitory control, working memory and cognitive flexibility. The analyzes showed differences between the athlete and non-athlete groups in the verbal-auditory inhibitory control, the Odd One Out working memory test and the Trail Making Test. In conclusion, soccer players have better inhibitory control, cognitive flexibility, and working memory than their peers of the same age and school class. This highlights the need for longitudinal studies to further investigate the importance of "higher level" cognitive functions for talent identification, talent development and planning of pedagogical processes in the context of football. Keywords: Executive function; Football; Motor learning; Children.
\end{abstract}

\section{Cite this article as:}

Sartori, R.F., Mezavilla, D., Nobre, G.C., \& Fonseca, R.P. (2020). Do executive functions discriminate sports performance in young soccer athletes? (in Portuguese). Journal of Human Sport and Exercise, 15(1 proc), S84S93. doi:https://doi.org/10.14198/jhse.2020.15.Proc1.09

\footnotetext{
Corresponding author. Pontifical Catholic University of Rio Grande do Sul, Porto Alegre (PUCRS), RS, Brazil 6681, Ipiranga Avenue, Building 80, 90619-900, Porto Alegre, RS Brazil.

E-mail: rodrigo.sartori@pucrs.br

Supplementary Issue: Rio 2016 Olympic Games Third Anniversary Special Edition. Olympic Studies Forum, 2-3 September 2019. Federal University of Sergipe, Aracaju, Brazil.

JOURNAL OF HUMAN SPORT \& EXERCISE ISSN 1988-5202

(c) Faculty of Education. University of Alicante

doi:10.14198/jhse.2020.15.Proc1.09
} 


\section{RESUMO}

As funções executivas são importantes para um bom desempenho no futebol, exigindo adaptação a situações em constante mudança do ambiente e rapidez na antecipação de suas ações, com consequente eficiência de planejamento. Em nosso estudo buscou-se comparar a capacidade de desempenho das funções executivas em jovens atletas de futebol e em um grupo de seus colegas de escola (não atletas), além de verificar qual domínio executivo é mais discriminativo. Participaram deste estudo um total de oitenta pré-adolescentes com idade de 11 e 12 anos divididos em 2 grupos. Foram avaliados os seguintes componentes; controle inibitório, memória de trabalho e flexibilidade cognitiva. Os resultados apontaram diferenças entre o grupo de atletas e não atletas no controle inibitório auditivo-verbal no teste Go-noGoApp, no teste de memória de trabalho Odd One Out e no teste de flexibilidade cognitiva com o teste Trail Making Test, com melhor desempenho para o grupo de atletas. Além disso, a função discriminante linear de Fisher também indicou que a variável da função executiva controle inibitório foi capaz de discriminar os grupos de atletas e não atletas. Em conclusão, atletas de futebol das categorias de base têm melhor desempenho de funções executivas do que seus colegas de mesma idade e classe escolar não atletas. Isso destaca a necessidade de estudos longitudinais e de intervenção para investigar mais a importância das funções cognitivas de "nível superior" como mais uma variável que possa auxiliar para a identificação de talentos e planejamento dos processos pedagógicos no contexto do futebol. A estimulação precoce-preventiva de funções executivas em atletas em formação pode contribuir para uma melhor performance motora e cognitiva de jogo no futebol. Palavras Chave: Funções executivas; Futebol; Aprendizagem motora; Crianças. 


\section{INTRODUÇÃO}

Na perspectiva de estudos interdisciplinares, cabe ressaltar que novos estudos na área da neuropsicologia creditam importante papel às estruturas neurais para compreender como estas se organizam a fim de auto ajustar-se nas dinâmicas situações do cotidiano das pessoas. Dentre essas situações cotidianas, encontrase 0 jogo de futebol, caracterizado por demandas cognitivas necessárias para realização de tomadas de decisão eficazes. Para tal, é necessário compreender que a relação entre aspectos biológicos e ambientais irão definir os processos cognitivos do comportamento humano e, dentre esses processos, encontram-se as funções executivas.

As funções executivas são formas de cognição que têm como função a percepção e atuação do sistema nervoso central na regulação do comportamento humano (Lezak, 1982; Diamond 2013) e, neste caso, incluise 0 comportamento motor (Leonard, 2016). Embora habilidades motoras e cognitivas sejam frequentemente estudadas separadamente, é crescente a compreensão da estreita relação entre esses domínios do desenvolvimento (Leonard; Hill, 2015). As funções executivas, tal como vêm sendo estudadas nas publicações sobre o desenvolvimento cognitivo, têm sido vinculadas às habilidades específicas de processamento de informações, divididas em 3 funções principais de acordo com Miyake et al., (2000): (1) memória de trabalho, definida como 0 armazenamento e a atualização das informações enquanto o indivíduo desempenha alguma atividade relacionada a elas; (2) controle inibitório, definido como a inibição da resposta prepotente ou automatizada quando o indivíduo está empenhado na execução de uma tarefa, e (3) flexibilidade mental, definida como a capacidade de mudar a postura de atenção e cognição entre dimensões ou aspectos distintos - mas relacionados - de uma determinada tarefa (Diamond, 2013; Miyake et al., 2000).

Em esportes de habilidade aberta, como o futebol, (Magill, 2003) o ambiente muda constantemente; por isso, jogadores devem processar uma grande quantidade de informações externas e tomar as ações apropriadas em um período curto (Sakamoto, 2018). As funções executivas, as quais regulam pensamentos e ações, são importantes para o desempenho bem-sucedido em esportes, principalmente em esportes coletivos como, por exemplo, o futebol, que exige rapidez, antecipação e adaptação a situações em constante mudança de seu meio (Verburgh, 2014).

Na medida em que as funções executivas são complexas, apresentando subdomínios e relações entre seus componentes, a avaliação neuropsicológica envolve tipos de procedimentos específicos para cada uma das funções (Pureza et al., 2013). A avaliação neuropsicológica das funções executivas tem despertado interesse dos pesquisadores por ser um procedimento de investigação das relações entre cérebro e comportamento, especialmente das disfunções cognitivas associadas aos distúrbios do sistema nervoso central (Hamdan; Pereira, 2009). Porém, apesar dos avanços na área de investigação neuropsicológica, não se conseguiu ainda decifrar o funcionamento de nosso cérebro e todos os processos cognitivos que envolvem as inúmeras operações e funções que se desempenha para a realização do comportamento motor e mais especificamente nas tarefas do esporte.

Pensando no contexto do futebol, atletas podem ser confrontados com situações de tomada de decisão a partir da imprevisibilidade do contexto do jogo. Cabe ressaltar que os comportamentos aprendidos anteriormente, os quais se tornaram mecânicos, não funcionam mais, e processos de controle adaptativo adicionais devem ser recrutados para atender às demandas de situações (Diamond, 2013). Nesse sentido, a nova natureza da situação exige que "descubramos o que fazer" por conta própria, de forma independente. Precisa-se criar uma resposta diferente e tomar uma decisão diferente a cada momento do jogo e nesse caso a avaliação neuropsicológica do esporte parece ser fator imprescindível no contexto esportivo, poisas 
situações de jogo exigem controle cognitivo de ordem superior, o que também pode ser chamado de manifestação dos componentes das funções executivas (Koziol \& Lutz, 2013).

A identificação e sobretudo a formação de talentosos jogadores de futebol é considerada de grande importância por muitos clubes profissionais. Essa identificação permite selecionar aqueles jogadores com potencial para atingir o nível superior e ao mesmo tempo o processo de formação dentro desta perspectiva, possibilita um maior investimento focado nos programas de desenvolvimento de atletas (Verburgh, 2013, 2014). Nesse sentido, uma estratégia eficaz é comparar atletas de futebol bem-sucedidos com grupos controles, a fim de determinar os fatores que parecem ser importantes para alcançar o sucesso ou variáveis que discriminem os promissores atletas. Por exemplo, alguns estudos na Holanda e no Japão (Huijgen, 2015; Sakamoto, 2018), ao comparar atletas de elite com amadores, identificaram que os grupos de elite apresentam melhor desempenho das funções executivas. Além disso, este estudo justifica-se pelo fato de não haver uma literatura no Brasil que discuta o impacto das funções cognitivas (executivas) para a formação de atletas no futebol. Assim sendo, buscou-se comparar a capacidade de desempenho das funções executivas em jovens atletas de futebol e em um grupo de seus colegas de escola (não atletas), além de verificar qual domínio executivo é mais discriminativo.

\section{MATERIAIS E MÉTODOS}

\section{Participantes}

Foram avaliados oitenta pré-adolescentes de 11 e 12 anos, do sexo masculino, separados em dois grupos. O primeiro grupo composto por atletas de futebol, de um clube profissional do Rio Grande do Sul - Brasil, e o segundo grupo composto por alunos de duas escolas públicas onde estes atletas estudam. Cada criança do grupo de atletas $(n=40$ meninos, Média $=8,74, D P=0,34)$ foi aleatoriamente pareada com uma criança do grupo de escolares ( $n=40$ meninos, Média $=8,94, D P=0,60)$. Os grupos foram semelhantes em relação à idade, série escolar e status socioeconômico da família (valores de $p \geq 0,05$ ). Crianças com dificuldades de aprendizagem no contexto escolar avaliado pelos pais e/ou professores ou diagnóstico médico de incapacidades e transtorno de déficit de atenção e hiperatividade (TDAH), investigado através da secretaria da escola, foram excluídas do presente estudo.

\section{Instrumentos}

Neste tópico, serão apresentados os instrumentos utilizados para avaliação das funções executivas nos grupos de atletas e não atletas.

O GonogoApp foi desenvolvido tendo como base a tarefa de inibição de comportamento, modulação e inibição de resposta denominada GONOGO (Nosek; Banaji, 2001). Os testes Go-No-Go App, visual-motor e auditivo-verbal, foram utilizados para avaliar o controle inibitório. $O$ aplicativo Go-No-Go é um teste que contém 60 números registrados em 2 tarefas que fornecem duas formas de estímulos (auditivo e visual) e duas formas de respostas (motor e verbal). As pontuações são compostas pelos erros (no visual motor, 0 indivíduo deve tocar a tela a cada número que aparece, exceto quando for o número 6 , para o qual não fará nenhum movimento; no auditivo verbal, deve falar "sim" a cada número falado pelo aplicativo, exceto também no número 6, no qual não deve falar nada) e gravadas no smartphone.

Na tarefa Odd One Out, para avaliação da memória de trabalho, é apresentada para as crianças uma quantidade crescente de informações para processar e, em seguida, questiona-se se algum aspecto particular do posicionamento espacial do objeto é lembrado (Henry, 2001). É um teste de span complexo, no qual os avaliados veem pequenas matrizes de três "caixas" com uma forma dentro de cada uma. Duas 
destas formas são sempre idênticas. Os avaliados têm que identificar a forma que não combina, memorizar sua localização em cada matriz e evocar a localização de todas as formas diferentes, na sequência correta. A evocação é feita a partir de matrizes em branco, ao final de cada bloco. 0 teste avalia a capacidade da memória operacional viso-espacial. 0 sujeito recebe 1 ponto para cada recordação (pontuação da memória de trabalho). Os números totais de tentativas corretas na tarefa serão utilizados na análise.

Para a avaliação da flexibilidade cognitiva, foi utilizado o teste de trilhas - Parte A e B (Montiel; Seabra, 2012). $O$ instrumento é dividido em duas partes. A parte A é voltada para a busca visual e composta por duas folhas (uma para letras e outra para números). Na primeira folha, são apresentadas 12 letras dispostas aleatoriamente de "A" a "M" (ausência da letra K). Nela, o sujeito deve ligar as letras em ordem alfabética. Na segunda folha, números de "1" a "12" estão dispostos aleatoriamente na folha, e o sujeito deve ligá-los em ordem crescente. Já na parte $B$, as letras (12 itens) e os números (12 itens) estão dispostos aleatoriamente, e o sujeito deverá ligá-los de maneira intercalada: primeiro, letra e, depois, número. A pontuação foi obtida pelo número de itens conectados corretamente em uma sequência ininterrupta na parte B durante 1minuto.

\section{Procedimentos}

O presente estudo foi aprovado pelo comitê de Ética e Pesquisa de uma Universidade. O clube e a escola concordaram em fazer parte do estudo através de reuniões com diretores e responsáveis. As avaliações foram todas feitas em ambiente fechado (salas), com os avaliados sentados, sem interferência externa, de forma individual. Antes de iniciar os testes, os alunos e atletas receberam uma breve explicação sobre os mesmos; cada aluno/atleta levou, em média, 20 minutos para fazer a bateria de testes. Todos os testes foram administrados e pontuados de acordo com os procedimentos recomendados nos manuais por dois profissionais treinados para realizá-los.

\section{Análise estatística}

A análise e a descrição dos resultados foram realizadas por meio de média e desvio padrão, frequência e percentual. Para avaliar as possiveis diferenças entre as variáveis de função executiva entre os atletas e não atletas e se essas variáveis discriminariam os grupos, foi realizada uma análise discriminante linear de Fisher. $O$ critério de Wilk-Lambda e o método stepwise foram empregados na estimação do modelo. 0 teste $\mathrm{M}$ de Box para igualdade das matrizes de covariância foi utilizado. Os valores de assimetria e curtose uni e multivariada foram utilizadas para analisar a normalidade da distribuição dos dados. Foram considerados valores maiores que 3 para assimetria e maiores que 7 para a curtose como violação severa à distribuição normal. 0 nível de $a<.05$ foi adotado.

\section{RESULTADOS}

$O$ teste $M$ de Box mostrou igualdade nas matrizes de covariância ( $M=42.027, F(1)=13.620, p=.061)$ e os valores de assimetria (entre .3 e 1.13) e curtose univariadas (.820 e -.503) e curtose multivariada (5.884) não mostraram violação à distribuição normal. Estes resultados dão suporte ao uso da análise discriminante. As análises mostraram diferenças entre o grupo de atletas e não atletas no controle inibitório auditivo-verbal (Wilks' $\Lambda=.806, F(1,78)=18.750, p<.001$ ), no teste de memória de trabalho OddOneOut (Wilks' $\Lambda=.904$, $F(1,78)=8.281, p=.005$ ) e no teste de flexibilidade cognitiva TMT- SQ (Wilks' $\Lambda=.902, F(1,78)=8.457, p$ $=.005)$. A tabela 1 apresenta as médias e desvio padrão das variáveis de função executiva de acordo com os grupos e em geral. 
Tabela 1. Médias e desvios padrão das variáveis de função executiva dos atletas, não atletas e do grupo em geral.

\begin{tabular}{|c|c|c|c|}
\hline \multirow{3}{*}{ Função executiva } & \multicolumn{2}{|c|}{ Grupos } & \multirow{3}{*}{$\begin{array}{l}\text { Geral } \\
M(d p)\end{array}$} \\
\hline & Atletas & Não atletas & \\
\hline & $M(d p)$ & $M(d p)$ & \\
\hline GNG-AV* & $.6(1)$ & $2.1(2)$ & $1.4(1.7)$ \\
\hline GNG-VM* & $1.6(1.5)$ & $2.15(1.7)$ & $1.9(1.6)$ \\
\hline ODD* & $13.9(5)$ & $10.8(4.5)$ & $12.4(5)$ \\
\hline TMT-N* & $13(4.6)$ & $19.3(10.2)$ & $16.1(8.5)$ \\
\hline TMT- L* & $15(5.4)$ & $21.9(11.6)$ & $18.4(9.6)$ \\
\hline TMT- SQ* & $17.1(4.8)$ & $14.1(4.4)$ & $15.6(4.8)$ \\
\hline
\end{tabular}

*Resultados GNG (erros); ODD ( acertos); TMT-N e L (tempo); TMT SQ ( número de sequencias corretas).

A função discriminante linear de Fisher indicou que a variável da função executiva controle inibitório auditivoverbal (coeficiente da função de discriminação $=.757$ ) foi capaz de discriminar os grupos atletas e não atletas; Wilks' $\Lambda=.723, X^{2}(2,77)=24.980, p<.001$. A tabela 2 apresenta a taxa de sucesso para a predição dos grupos pela função discriminante. A análise mostrou que, em geral, o modelo classificou corretamente $68.8 \%$ dos casos.

Tabela 2. Taxa de sucesso para a previsão de participação no grupo para a análise discriminante.

\begin{tabular}{lccc} 
Grupos & \multicolumn{3}{c}{ Previsão de associação ao grupo n(\%) } \\
\cline { 2 - 4 } Não atletas & Não atletas & Atletas & Total \\
Atletas & $22(55)$ & $18(45)$ & $40(100)$ \\
\hline
\end{tabular}

$68.8 \%$ dos casos originais classificados corretamente.

\section{DISCUSSÃO}

O presente estudo, que teve como objetivo comparar as funções executivas em atletas e não atletas de futebol, mostrou que os atletas superaram os alunos não atletas em todas as variáveis investigadas: controle inibitório, memória de trabalho e flexibilidade cognitiva. Uma revisão sistemática que foi realizada recentemente confirma esta mesma tendência onde observou-se que os atletas adolescentes que treinavam futebol em clubes profissionais apresentaram melhores resultados nos testes de FE do que os adolescentes que treinavam em clubes amadores (Paiano et al 2019).

Um primeiro ponto de discussão pode ser apresentado no sentido de identificar se a prática do futebol pode ter sido o efeito da melhor capacidade das funções executivas no grupo de atletas por conta das características de demanda cognitiva que o jogo apresenta. Por outro lado, é importante apresentar hipóteses relacionadas ao perfil cognitivo desses jovens, compreendendo que algumas crianças, no processo de formação esportiva, apresentam características pessoais pré-determinadas para 0 desenvolvimento das capacidades supracitadas. Dessa forma, cabe discutir cada um dos componentes das funções executivas avaliados nos dois grupos para melhor entender as relações destes componentes com a prática do futebol.

Os resultados do nosso estudo apontam o controle inibitório como variável que pode discriminar grupos de atletas e não atletas de futebol. Este componente refere-se à capacidade de inibir respostas e estímulos, especialmente os que já estão em curso (Barkley, 2001). Esse controle está relacionado, por exemplo, à 
capacidade de suprimir uma resposta mesmo quando existem estímulos internos e externos gratificantes, os quais, dessa forma, envolvem a capacidade de resistir às tentações e não agir de maneira impulsiva ou prematura (Diamond, 2000). Essas características parecem estar presentes em várias situações do esporte, especificamente no futebol, no qual no mesmo momento em que a equipe está em um momento ofensivo, existe a necessidade de inibir este comportamento na medida em que ocorre uma perda de posse de bola, o que é denominado de transições ofensivas e defensivas (Da Costa et al 2009). Da mesma forma, as funções executivas parecem estar associadas ao controle das ações técnicas como o passe, drible, finalização e também das mais situações mais complexas, tomando as decisões corretas como acertar 0 tempo certo da finalização ao gol, não precipitando a ação, ou controlar a abordagem da marcação em situações (1x1) para não fazer faltas desnecessárias.

O grupo de atletas mostrou um controle maior de suas ações nas tarefas avaliadas, tendo, assim, uma capacidade e controle maiores de inibir respostas quando comparados ao grupo de não atletas. Esses resultados vêm ao encontro de um estudo no qual jogadores de futebol profissional obtiveram significativamente melhores resultados nas tarefas de controle inibitório quando comparado aos atletas amadores (Verburgh; 2014). Os dados desse estudo mostraram que, apesar dos profissionais levarem um tempo maior para executar a tarefa, seus acertos foram maiores que os dos amadores devido à capacidade de controlar melhor suas decisões. O controle inibitório em jovens atletas de futebol de elite parece ser mais eficiente quando comparado a jovens atletas de futebol amador (Huijgen, 2015). Nesse sentido, percebe-se que a capacidade de inibir rapidamente as respostas motoras durante habilidades relacionadas ao futebol parece ser um fator determinante para ser um jovem jogador de futebol bem-sucedido.

O presente estudo também avaliou e comparou o componente memória de trabalho entre o grupo de atletas e não atletas apontando melhores resultados para os jovens praticantes de futebol. A memória de trabalho é um sistema temporário e ultrarrápido de armazenamento de informações, o qual permite o monitoramento e o manejo destas informações (Baddeley, 2003) e relaciona-se à retenção das mesmas na mente e à possibilidade de trabalhar mentalmente com essas memórias, relacionando uma coisa à outra ou usando pistas para resolver um problema (Diamond, 2013). Durante o jogo de futebol, uma série de ações são realizadas e dependem da capacidade da memória de trabalho, como, por exemplo, visualizar imagens de situações do jogo e conseguir reter essas informações para, na sequência do lance, tomar decisões mais eficazes.

Neste esporte com características de imprevisibilidade no qual atletas precisam se adaptar e interagir com o contexto do jogo todo o tempo, a memória de trabalho tem um papel importante pela possibilidade de associar as sequências de cenas do jogo para tomar decisões eficazes. Na memória de trabalho visoespacial, as informações são mantidas na mente durante o jogo, pois os atletas precisam processar inúmeras situações: tempo de bola, instruções do treinador, posição em campo, entre outras (Alesi; 2016). Essas capacidades cognitivas ressaltam a importância do estudo deste tema tanto na captação como nos processos de intervenção em programas de treinamento do futebol. Ao avaliar as funções executivas em atletas de futebol de elite e amadores; os resultados indicaram diferenças na memória de trabalho visoespacial entre os grupos, a favor do grupo de elite (Verburgh, 2014). Esses resultados podem estar relacionados à maior exigência dessas funções cognitivas durante treinos e jogos. É importante mencionar que os achados não permitem concluir sobre a capacidade de predição desses dados para formação de atletas profissionais de futebol (Verburgh, 2014). Sugere-se, nesse sentido, a realização de estudos longitudinais para acompanhar o desenvolvimento dos jovens até o processo de abandono ou transição para a categoria profissional. 
Com relação a flexibilidade cognitiva também encontramos diferenças entre os grupos em favor do grupo de atletas. Esse componente das funções executivas diz respeito à capacidade do indivíduo de modular seus comportamentos e, assim, adaptar-se a diferentes regras ou exigências de determinada tarefa (Leonard et al., 2015). Também pode ser referida à capacidade de mudar o curso de ações e/ou pensamentos de acordo com a exigência do ambiente (Dos Santos Assef; Seabra; Capovilla, 2007). Um estudo holandês, (Huijgen, 2015) comparou atletas de futebol juvenil e de profissionais, também investigaram a capacidade de flexibilidade cognitiva com o mesmo instrumento de avaliação (Trail Making Test) utilizado em nosso estudo. Os resultados desse estudo permitem mostrar que há diferença entre os grupos, a favor dos atletas profissionais. 0 melhor desempenho de atletas de melhor performance é explicado através das demandas e exigências a que esses atletas foram submetidos ao longo da carreira (Huijgen, 2015). Provavelmente o nível de complexidade da tarefa relacionado ao jogo de futebol pode contribuir para um melhor desenvolvimento das funções executivas e que melhores indicadores de FE foram encontrados em praticantes mais habilidosos de futebol, o que poderia estar associado a outros esportes (Paiano et al 2019). Nesse sentido, atletas profissionais de voleibol apresentam melhor desempenho da flexibilidade cognitiva quando comparados a um grupo de atletas de categorias de base do mesmo esporte (Montuori, 2019).

\section{CONCLUSÃO}

Em conclusão, jogadores de futebol das categorias de base têm melhor controle inibitório, flexibilidade cognitiva e memória de trabalho, do que seus colegas de mesma idade e classe escolar. O componente de controle inibitório pode discriminar um grupo de atletas de um grupo de não atletas nesta amostra. No entanto, cabe ressaltar que o processo de formação esportiva é multifatorial e deve ser pensando na perspectiva de um fenômeno complexo e sujeito a outras variáveis que devem ser também valorizadas. Com relação ao que trata das funções executivas no futebol, estes dados destacam a necessidade de estudos longitudinais e de intervenção para investigar a importância das funções cognitivas de "nível superior" para identificação de talentos, desenvolvimento de talentos e planejamento dos processos pedagógicos no contexto do futebol.

Pode-se inferir ainda que a relação entre funções executivas e desempenho no futebol é um caminho promissor de pesquisa para identificar possíveis características cognitivas que favoreçam o desempenho esportivo e como essas funções impactam no desempenho técnico e tático em situações específicas. 0 futebol, assim como o esporte de maneira geral, parece melhorar a capacidade de adaptação, planejamento, resolução de problemas e até mesmo o controle e inibição de ações.

\section{REFERÊNCIAS}

Alesi, Marianna et al. (2016). Improving children's coordinative skills and executive functions: the effects of a football exercise program. Perceptual and motor skills, v. 122, n. 1, p. 27-46, 2016. https://doi.org/10.1177/0031512515627527

Baddeley, A., \& Larsen, J. D. (2003). The disruption of STM: A response to our commentators. The Quarterly Journal of Experimental Psychology Section A, 56(8), 1301-1306. https://doi.org/10.1080/02724980343000530

Capovilla, A. G. S., dos Santos Assef, E. C., \& Cozza, H. F. P. (2007). Avaliação neuropsicológica das funções executivas e relação com desatenção e hiperatividade. Avaliação psicológica, 6(1), 51-60. https://doi.org/10.11606/d.5.2009.tde-09022010-171726 
Da Costa, I. T., da Silva, J. M. G., Greco, P. J., \& Mesquita, I. (2009). Princípios táticos do jogo de futebol: conceitos e aplicação. Motriz. Journal of Physical Education. UNESP, 657-668.

Diamond, A.(2013). Executive functions. Annual Review of Psychology, v.64, p.135-168.

Dos Santos Assef, E. C., Capovilla, A. G. S., \& Capovilla, F. C. (2007). Avaliação do controle inibitório em TDAH por meio do teste de geração semântica. Psicologia: teoria e prática, 9(1), 61-74.

Hamdan, A. C., \& de Almeida Pereira, A. P. (2009). Avaliação neuropsicológica das funções executivas: considerações metodológicas. Psicologia: Reflexão e crítica, 22(3), 386-393. https://doi.org/10.1590/s0102-79722009000300009

Hanna-Pladdy, B. (2007). Dysexecutive syndromes in neurologic disease. Journal of Neurologic Physical Therapy, v. 31, n. 3, p. 119-127. https://doi.org/10.1097/npt.0b013e31814a63c2

Henry, L. (2001). How does the severity of a learning disability affect working memory performance? Memory, v. 9, n. 4-6, p. 233-247. https://doi.org/10.1080/09658210042000085

Huijgen, Barbara $\mathrm{CH}$ et al. (2015). Cognitive functions in elite and sub-elite youth soccer players aged 13 to 17 years. PloS one, v. 10, n. 12, p. e0144580. https://doi.org/10.1371/journal.pone.0144580

Koziol, L. F., \& Lutz, J. T. (2013). From movement to thought: the development of executive function. Applied Neuropsychology: Child, 2(2), 104-115. https://doi.org/10.1080/21622965.2013.748386

Leonard, H. C. (2016). The impact of poor motor skills on perceptual, social and cognitivedevelopment: The case of developmental coordination disorder. Frontiers in Psychology, 7(MAR), 1-4. https://doi.org/10.3389/fpsyg.2016.00311

Leonard, H. C., \& Hill, E. L. (2015). Executive Difficulties in Developmental Coordination Disorder: Methodological Issues and Future Directions. Current Developmental Disorders Reports, 2(2), $141-$ 149. https://doi.org/10.1007/s40474-015-0044-8

Lezak, MD (1982). O problema de avaliar funções executivas. International journal of Psychology , 17 (1-4), 281-297.

Magill, R. A. (2008). Aprendizagem motora: conceitos e aplicações. Edgard Blücher.

Miyake, A., Kominami, H., Tatsuoka, H., Kuwabara, H., Nakanishi, Y., \&Hatanaka, Y. (2000). Growth of epitaxial ZnO thin film by oxidation of epitaxial ZnS film on Si (111) substrate. Japanese Journal of Applied Physics, 39(11B), L1186. https://doi.org/10.1143/jiap.39.11186

Montiel, J. M., \& Seabra, A. G. (2012). Teste de Trilhas-partes A e B. Avaliação neuropsicológica cognitiva: atenção e funções executivas, 1, 79-85.

Montuori, S et al. (2019). Executive functioning profiles in elite volleyball athletes: Preliminary results by a sport-specific task switching protocol. Human movement science, 63, 73-81. https://doi.org/10.1016/j.humov.2018.11.011

Nosek, B. A., \&Banaji, M. R. (2001). The go/no-go association task. Social cognition, 19(6), 625-666. https://doi.org/10.1521/soco.19.6.625.20886

Paiano, R., Amaro, A. S., Garcia, F., Ferreira, R. C. T., Ressurreição, K. S., \& Carreiro, L. R. R. (2019). Futebol e funções executivas: um estudo de revisão. Cadernos de Pós-Graduação em Distúrbios do Desenvolvimento, 19(1). https://doi.org/10.5935/cadernosdisturbios.v19n1p81-97

Pureza, J. R., Jacobsen, G. M., Grassi-Oliveira, R., \& Fonseca, R. P. (2011). Relationships between executive functions tasks in late childhood. Psychology and Neuroscience, 4(3), 369-376. https://doi.org/10.3922/i.psns.2011.3.010

Sakamoto, Shota et al.(2018). Possible requirement of executive functions for high performance in soccer. PloS one, v. 13, n. 8, p. e0201871. https://doi.org/10.1371/journal.pone.0201871

Verburgh, L et al. (2014). Executive functioning in highly talented soccer players. PloS one, 9(3), e91254. https://doi.org/10.1371/journal.pone.0091254

Verburgh, Lot et al. (2014). Executive functioning in highly talented soccer players. PloS one, v. 9, n. 3, p. e91254. https://doi.org/10.1371/journal.pone.0091254 
Verburgh, Lot et al.(2016). Do elite and amateur soccer players outperform non-athletes on neurocognitive functioning? A study among 8-12 year old children. PloS one, v. 11, n. 12, p. e0165741. https://doi.org/10.1371/journal.pone.0165741

Vestberg, Torbjörn et al. (2017). Core executive functions are associated with success in young elite soccer players. PloS One, v. 12, n. 2, p. e0170845. https://doi.org/10.1371/journal.pone.0170845

\section{(c) (i) (3)}

This work is licensed under a Attribution-NonCommercial-NoDerivatives 4.0 International (CC BY-NC-ND 4.0). 